\title{
A MODIFIED ENERGY-BASED MODEL FOR DESCRIBING WEAR PROCESSES APPLIED TO AN INTERNAL COMBUSTION ENGINE
}

\author{
J. SEQUARD-BASE ${ }^{1}$, C. LENAUER ${ }^{1}$, V. LAZAREV ${ }^{2}$, K. GAVRILOV ${ }^{3}$, A. DOIKIN ${ }^{3}$ \& G. VORLAUFER ${ }^{1}$ \\ ${ }^{1} \mathrm{AC}^{2} \mathrm{~T}$ Research $\mathrm{GmbH}$, Austria. \\ ${ }^{2}$ Department of ICE, South Ural State University, Russia. \\ ${ }^{3}$ Department of Automobile Transport, South Ural State University, Russia.
}

\begin{abstract}
To describe and predict wear in a tribosystem, theoretical wear formulas and empirical models exist. Most of the existing formulations have a short range of validity and can only be applied for a specific contact situation. In this paper, the aim is to investigate a specific technical application of a tribocontact in an internal combustion engine. In particular, the contact between a piston skirt and a cylinder liner is experimentally simulated using a linear reciprocating tribometer and original engine parts, under closeto-reality loading conditions. The experimental findings will be analysed with a wear model that is most applicable to the actual tribosystem. This wear model, which is based on a combination of energy theories and a molecular mechanics approach, will be extended in the paper in order to add surface topography relevant parameters. The modified wear model is capable of combining the prediction of wear volume loss with the theory of fatigue and can be applied to any kind of tribosystems suffering damage due to reciprocating relative motion. For comparison with classical wear models, an empirical power law - including Archard as a special case - is shown. Using the measurement results of the tribometer, the parameters that are specific to the aforementioned wear model are determined. Furthermore, the applicability of the used model to describe the wear processes in this specific tribosystem will be discussed.
\end{abstract}

Keywords: friction energy, internal combustion engine, tribometer test, wear, wear modelling.

\section{INTRODUCTION}

Describing and predicting the phenomenon of wear in a tribosystem is a fundamental task of tribology. This seemingly simple question has led to more than 300 different formulas and models during the last 80 years [1], which claim to work properly for some specific contact situations. In principle, there are two possibilities of approaching this challenge.

The first option is establishing a theoretical wear model, starting at micro- and even sub-micromechanical properties of the materials and the contact situations on the atomic length scale, in order to calculate the wear based on physical laws. This method is preferable because it describes an explicit relationship between material properties and contact parameters, making it possible to obtain a fundamental understanding of the processes in the tribocontact and showing which physical or chemical factors are pivotal for the loss of material during the frictional interaction.

The second option is to use the results of well-documented experiments and build up a parameter model, which is capable of fitting the wear curves according to the measurement outcomes. A drawback of the latter methods is that the used parameters per se have no direct physical denotation, and so it is difficult to gain a deeper insight into the actual processes. Moreover, the considerable number of constants used in some of these formulas makes them cumbersome to use [2].

The main problem of all wear equations is that there is no unique wear process, and so many equations just focus on one specific wear mechanism. For this reason, a lot of 
methods are not suited for the use in technical applications, especially when several different wear mechanisms and regimes are prevalent in the tribosystem of interest. Furthermore, a look into literature shows that a general valid formula describing wear has not yet been established [3].

The first intention of this paper is to develop a modified version of an alternative approach in wear theory based on an energy balance formulation by Fleischer [4] and Lazarev et al. [5].

In a second step, this modified model is used to describe a widespread technical application of a tribosystem according to wear. The investigated contact is between the piston skirt and the cylinder liner of an internal combustion engine, which is one of the major contributors to the failure of automotive engines [6]. When taking into account the total number of vehicle production of more than 87 million in 2013, the significance of this tribocontact to world economy and society is clear [7].

In the third step, the description of the modified wear model will be compared to a classical parameter wear model according to the second category mentioned above.

\section{WEAR MODELS}

2.1 Declaration of variables and parameters

$W_{\mathrm{R}} \quad$ total friction energy of one stroke

$F_{\mathrm{t}} \quad$ tangential force

$\Delta s \quad$ sliding distance of one stroke

$\mu \quad$ coefficient of friction

$F_{\mathrm{n}} \quad$ normal force

$A_{\mathrm{N}} \quad$ nominal contact area

$A_{\mathrm{R}} \quad$ real contact area of the softer material. Note: different from the nominal contact area $A_{\mathrm{N}}$

$p_{\mathrm{A}} \quad$ nominal contact pressure

$W_{\mathrm{Th}} \quad$ thermal energy, which ultimately results in the main part of the total friction energy of one stroke

$W_{\mathrm{D}} \quad$ 'stored' deformation energy, which constitutes a minor part of the total friction energy of one stroke

$\rho \quad$ coefficient of accumulation of friction energy; it indicates which fraction of the total friction energy is 'stored' in lattice defects of the contact partners

$\Delta V \quad$ mean worn material volume

$\Delta h \quad$ mean height of the worn material

$V_{\mathrm{W}} \quad$ real worn material volume and therefore equivalent to the volume of elastic deformations

$\Delta H \quad$ height of the worn material

$I_{\mathrm{h}} \quad$ height of worn material layer divided by the sliding distance, called linear integral intensity of wear

$\theta \quad$ wear rate representing wear height per sliding time

$\omega \quad$ energy density

$\omega^{*} \quad$ critical energy density; it represents the energy density when the material strength is reached and particle separation takes place

$t \quad$ sliding time

$v \quad$ sliding velocity

$\sigma \quad$ real stress 
E Young's modulus of the softer contact partner

$\sigma_{\mathrm{B}} \quad$ true fracture stress

$H_{\mathrm{B}} \quad$ Brinell hardness

$W^{*}$ D critical 'stored' deformation energy at which particle separation takes place

$n_{\mathrm{K}} \quad$ critical number of strokes after which wear particles are produced

$\eta_{\text {As }} \quad$ asperity density of the softer tribopartner's surface

$N_{\text {As }} \quad$ total number of asperities of the softer tribopartner's surface

$n_{\mathrm{M}} \quad$ number of 'macroscopic' strokes

$n_{\mathrm{LC}} \quad$ number of 'macroscopic' load cycles

\subsection{Modified energy-based model}

In order to acquire a general description of the wear process, covering different wear mechanisms and regimes, one possibility is to focus on the energy transduced within a tribocontact. According to literature, these kinds of models are capable of giving more stable results than other methods based on classical approaches like Archard [8,9].

The principle of the model discussed in this paper, which is influenced by the ideas of Fleischer [4], is that due to the relative movement of the involved bodies, friction energy will be transported into the contact. In general, each body can 'store' a specific amount of energy in the surface layer until it breaks off, generating a new wear particle. In most cases, the friction energy first causes elastic deformations in the contacting surfaces, and after a period of relaxation the main part of this energy dissipates as heat into both tribopartners. Only a small part of the energy causes lattice defects during the deformations and will be 'stored'. After a critical number of load cycles, and therefore successively increasing amounts of 'stored' energy, the lattice defects are large enough to break off a small part of the surface, constituting wear.

\subsubsection{The basics of the model}

The total friction energy of one load cycle is the product of the tangential force and the sliding distance, and can be represented by

$$
W_{\mathrm{R}}=F_{\mathrm{t}} \Delta s
$$

Using the tangential force

$$
F_{\mathrm{t}}=\mu F_{\mathrm{n}}
$$

and the real stress (dividing the normal force $F_{\mathrm{n}}$ by the real contact area $A_{\mathrm{R}}$ )

$$
\sigma=\frac{F_{\mathrm{n}}}{A_{\mathrm{R}}},
$$

the friction energy of one stroke can be written as

$$
W_{\mathrm{R}}=\mu \sigma A_{\mathrm{R}} \Delta s .
$$

Both contact partners are exposed to this friction energy, which, at the first moment of contact interaction, is totally consumed by elastic mechanical deformations. After a certain relaxation time, the majority of the deformations will recover, in analogy to a damped harmonic oscillator. The kinetic energy due to these oscillations of the molecules in the contact layer will dissipate as thermal energy $W_{\mathrm{Th}}$ into both tribopartners. 
A small part of the total friction energy $W_{\mathrm{R}}$ will be stored in lattice defects. This small stored energy part is called $W_{\mathrm{D}}$ in the following.

The amount of the friction energy, which is not stored consists primarily of thermal energy $W_{\text {Th }}$ but could also include electronic excitations. The thermal energy could also initiate chemical reactions. For the following model, electronic excitation as well as tribochemical surface modifications are not considered.

After the relaxation time, the energy balance of one stroke can be written as

$$
W_{\mathrm{R}}=W_{\mathrm{Th}}+W_{\mathrm{D}} .
$$

It will be assumed that the tribocontact consists of two materials with different elastic and plastic material properties. The consequence of this assumption is that the stored elastic deformation energy in eqn (5) is primarily absorbed by the softer partner, whereas the thermal energy will be dissipated into both. In the following calculation, the real contact area of the softer material is equal to the real total contact area.

Furthermore, a proportional relationship between the total friction energy and the stored elastic deformation energy is assumed, with $\rho$ as the so-called 'coefficient of accumulation of friction energy' [4]:

$$
W_{\mathrm{D}}=\rho W_{\mathrm{R}} .
$$

With the assumption that by just one stroke the release of some wear is possible, this associated wear volume in the tribocontact can be represented as

$$
\Delta V=A_{\mathrm{R}} \Delta h,
$$

which includes the real contact area of the softer material $A_{\mathrm{R}}$ and the mean depth of the worn layer $\Delta h$.

A key parameter in the energy-based approach is the friction energy density $\omega$, which is generally determined by the actually stored part of the friction energy, divided by the volume absorbing this amount of energy. Particle separation from the contact layer takes place when the density of the stored energy, caused by former elastic deformations, reaches the critical value $\omega^{*}$.

For a single stroke, the total stored energy $W_{\mathrm{D}}$ is present within the mean worn material volume $\Delta V$. Note: $\Delta V$ is a statistical mean value and should not be mistaken for the real volume $V_{\mathrm{w}}$ of a wear particle

$$
\omega^{*}=\frac{W_{\mathrm{D}}}{\Delta V}=\frac{F_{\mathrm{t}} \Delta s \rho}{A_{\mathrm{R}} \Delta h}=\frac{\mu \sigma \Delta s \rho}{\Delta h} .
$$

Using the definition of the linear integral intensity of wear, which represents the wear height per sliding distance

$$
I_{\mathrm{h}}=\frac{\Delta h}{\Delta s}
$$

and inserting eqn (9) into eqn (8) leads to

$$
I_{\mathrm{h}}=\frac{\wp}{\omega^{*}} .
$$


A commonly used quantity for comparing theoretical results with experimental outcomes using the sliding velocity $v$ is the wear height per sliding time, called wear rate:

$$
\theta=\frac{\Delta h}{\Delta t}=\frac{\mu \sigma \rho}{\omega^{*}} v .
$$

Considering one stroke, the total friction energy $W_{\mathrm{R}}$ is acting on the volume $V_{\mathrm{W}}$, which is the volume that is elastically deformed. Lattice defects can occur in this volume alone, and therefore, this volume is the only volume released by wear if enough energy is stored in the lattice defects. The energy density $\omega$ can be expressed either by the total friction energy $W_{\mathrm{R}}$ divided by the worn volume $V_{\mathrm{w}}$, or by using the integral of Hooke's law:

$$
\omega=\frac{W_{\mathrm{R}}}{V_{\mathrm{W}}}=\frac{\sigma^{2}}{2 E} .
$$

To calculate the stress $\sigma$, it is necessary to estimate the real contact area. One possibility to achieve the real contact area is to use a mechanical FEM analysis of the contacting surfaces. Later on, a simplified model to estimate the real contact area will be used.

Assuming that in case of material destruction the real stress becomes the real fracture stress $\sigma_{\mathrm{B}}$, and again using Hooke's law, the critical energy density using the critical energy $W^{*}$ D can be expressed analogously

$$
\omega^{*}=\frac{W_{\mathrm{D}}^{*}}{V_{\mathrm{W}}}=\frac{\sigma_{\mathrm{B}}^{2}}{2 E} .
$$

Using eqns (12) and (13), it follows that

$$
W_{\mathrm{D}}^{*}=\frac{\sigma_{B}^{2}}{\sigma^{2}} W_{\mathrm{R}} .
$$

Note: Similar to Archard's law, the wear-related properties are described with a parameter acting perpendicular to the boundary layer, and not tangentially. However, the microscopic treatment of friction and wear mainly involves asperities that are perpendicularly orientated to the boundary zone. These asperities show bending movements consisting of tensile and compressive loadings. Therefore, the stress parameters $\sigma$ and $\sigma_{\mathrm{B}}$ are involved.

Inserting eqn (13) into eqns (10) and (11) completes the basics of the model

$$
\begin{gathered}
I_{\mathrm{h}}=\frac{\mu \sigma \rho}{\omega^{*}}=\frac{2 \mu \sigma E}{\sigma_{\mathrm{B}}^{2}} \rho, \\
\theta=\frac{\Delta h}{\Delta t}=\frac{2 \mu \sigma E v}{\sigma_{\mathrm{B}}^{2}} \rho .
\end{gathered}
$$

Qualitatively, these equations show that the amount of wear is higher if the stress $\sigma$ is higher or if the material-dependent $\sigma_{\mathrm{B}}$ is lower. Furthermore, a high Young's modulus is equivalent to a 'stiffer' material. Considering an asperity-asperity interaction, a 'stiffer' asperity is easier to break off than a 'softer' and more elastic one, and therefore a higher Young's modulus leads to a higher wear. Equations (15) and (16) are independent of the number of strokes. 


\subsubsection{Model extensions}

Fatigue phenomena of contacting materials during tribological processes occur by the change of their mechanical properties resulting in decreasing material strength. This is caused by the successively increasing stored energy in the form of lattice defects. The energy accumulated by the contact layer for several strokes $n$ is

$$
n W_{\mathrm{D}}=n \rho W_{\mathrm{R}} .
$$

In general, a critical number of strokes $n_{\mathrm{K}}$ is necessary to release a certain amount of wear. This minimal amount of wear material is the volume $V_{\mathrm{W}}$. In other words: if $n<n_{\mathrm{K}}$, theoretically no wear is released (Fig. 1).

Considering the hypothetical wear volume of one stroke $\Delta V$, the minimal volume for a released wear material of $V_{\mathrm{W}}$ is

$$
V_{\mathrm{W}}=n_{\mathrm{K}} \Delta V
$$

Assuming that the accumulated lattice defects are responsible for fraction, the energy balance during fracture of the contact layer can be represented as

$$
W_{\mathrm{D}}^{*}=n_{\mathrm{K}} W_{\mathrm{D}},
$$

where $\mathrm{W}^{*}{ }_{\mathrm{D}}$ denotes the critical energy in the volume $V_{\mathrm{W}}$. The critical energy density $\omega^{*}$ can be written, based on eqn (13), as

$$
\omega^{*}=\frac{W_{\mathrm{D}}^{*}}{V_{\mathrm{W}}}=\frac{\left(\frac{\sigma_{\mathrm{B}}}{\sigma}\right)^{2} W_{\mathrm{R}}}{V_{\mathrm{W}}}=\frac{n_{\mathrm{K}} \rho W_{\mathrm{R}}}{V_{\mathrm{W}}} .
$$

The basic stressed elements in boundary layers concerning friction and wear are asperities. In order to include the microscopic point of view, the critical number of strokes $n_{\mathrm{K}}$ must be distributed over the total number of all involved asperities $N_{\mathrm{As}}$. Therefore,

$$
n_{\mathrm{K}}=n_{\mathrm{M}} N_{\mathrm{As}},
$$

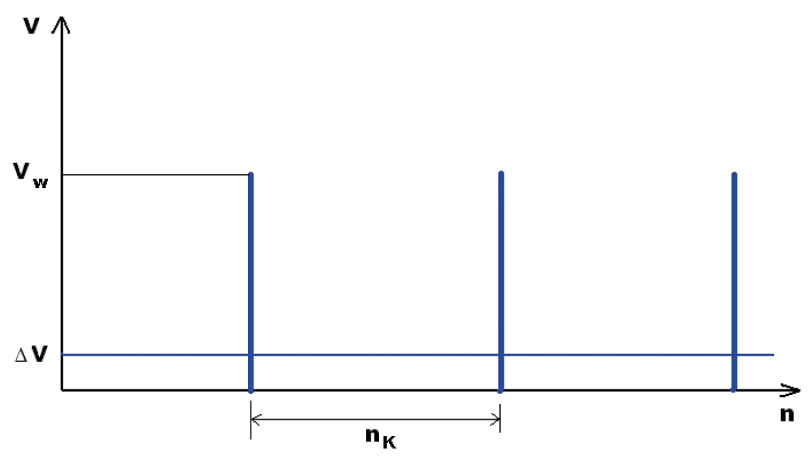

Figure 1: Illustration of the relationship between $\Delta V, V_{\mathrm{W}}$ and the critical number of strokes $n_{\mathrm{K}} \cdot \Delta V$ is the mean value and represents the hypothetical volume of wear for each single stroke. The true released volume of wear after the critical number of strokes is $V_{\mathrm{W}}$. 
with $n_{\mathrm{M}}$ being the number of macroscopic strokes seen in a reciprocating contact. Combining the right-hand side of eqns (20) and (21), the number of necessary macroscopic strokes will be

$$
n_{\mathrm{M}}=\frac{\left(\frac{\sigma_{\mathrm{B}}}{\sigma}\right)^{2}}{\rho N_{\mathrm{As}}} .
$$

Assuming low normal forces, a well-known approximation exists [10] concerning the relationship between the normal force $F_{\mathrm{n}}$, the real interacting surface $A_{\mathrm{R}}$ and the stress $\sigma$. This approximation expresses a proportional relation between $F_{\mathrm{n}}$ and $A_{\mathrm{R}}$. See eqn (3) with constant stress $\sigma$. Using the density of asperities $\eta_{\mathrm{As}}$ in eqn (22) leads to:

$$
n_{\mathrm{M}}=\frac{\sigma_{\mathrm{B}}^{2}}{\rho \sigma \eta_{\mathrm{As}} F_{\mathrm{n}}} .
$$

It should be mentioned that the model is not valid for the 'running-in' phase of a tribocontact. Therefore, $n_{\mathrm{M}}$ is the number of macroscopic strokes in the constant wear regime. Furthermore, it should be kept in mind that theoretically the conditions (i.e. the actual stored energy in form of lattice defects) are the same for all asperities representing the tribocontact. In reality, different asperities are subjected to different conditions, and therefore, each stroke produces a small amount of wear in different independent asperities. From a statistical point of view, this means that the amount of wear is represented by the volume $\Delta V$.

Checking the model is done by using the right-hand side of the macroscopic eqn (20)

$$
\omega^{*}=\frac{n_{\mathrm{K}} \rho W_{\mathrm{R}}}{V_{\mathrm{W}}}=\frac{n_{\mathrm{K}} \rho \mu F_{\mathrm{n}} \Delta s}{A_{\mathrm{R}} \Delta H} .
$$

Keeping in mind that

$$
\Delta H=n_{\mathrm{K}} \Delta h
$$

is the total height of wear after $n_{\mathrm{K}}$ load cycles, using eqn (3) for stress and eqn (13) for $\omega^{*}$, so eqn (24) can be straightforwardly transformed to the basic eqn (15) showing the consistence of the extended model.

Equations (15) and (16) represent a modified version of the energy-based approach, introduced by Fleischer and Kragelsky, correlating the unknown quantity of coefficient of accumulation of energy with parameters, which are accessible in an enhanced experimental tribometer set-up. Therefore, the first step of the experimental validation is to check the evolution of the coefficient of energy accumulation under varying loading conditions and sliding velocities.

\subsection{General power law of wear}

In the pioneering work of wear prediction, Holm and Archard assumed that the wear rate depends on the contact pressure $p$, and the hardness $H_{\mathrm{B}}$ of the material for adhesive mechanisms [11]:

$$
\theta=K \frac{p v}{H_{\mathrm{B}}}
$$


Experimental research showed that, despite its great popularity, not all technical tribosystems can be properly described using Archard's approach.

Recently, an extended approach was made that still depends on the contact pressure and the velocity, but generally in a non-linear way, which leads to the following equation:

$$
\theta=K_{\omega} p_{\mathrm{A}}^{a} v^{\beta}
$$

with the wear coefficient $K_{\omega}$ and the parameters $\alpha$ and $\beta$, which depend on material properties, contact conditions, temperature, etc. [12]. Archard's law is then a special case of the general formulation in eqn (27), with $K_{\omega}=K / H_{B}$, and a linear pressure and velocity dependence.

\section{EXPERIMENTAL INVESTIGATIONS}

\subsection{Measurement set up}

For the experimental investigation, test samples of piston skirt and cylinder liner fragments from internal combustion engines (see Fig. 2a), made of aluminium alloy ( $84 \mathrm{wt} \% \mathrm{Al}$ and $8 \mathrm{wt} \% \mathrm{Si}$ ) and cast-iron, respectively, were chosen and prepared in accordance with the requirements imposed by the tribotechnical instrumentation. For the sake of better reproducibility and comparability of the test results, the sample preparation included the application of a well-defined synthetic roughness of a honed structure with $S_{\mathrm{q}}$ value of approximately $3 \mu \mathrm{m}$.

The specimens were tested in a tribometer (SRV4 ${ }^{\circledR}$, Optimol Instruments) providing a linear oscillating relative movement. Such a tribometer platform allows an analysis of the impact of individual tribological parameters, like contact type, load, sliding velocity and lubrication conditions, independent of each other under laboratory conditions.

To mount the piston skirt and cylinder liner samples in the test chamber of the tribometer, a specially designed sample holder was used. This was necessary because, due to the specific contact geometry of the type block-on-plate, a very precise parallel alignment of the two specimens has to be guaranteed to avoid edge-running effects. Every test was run with Fresh Liqui Moly 5W30 Top Tec 4300 engine oil.

In combustion engines, the piston skirt is exposed to a wide spectrum of tribological conditions. The most challenging regions for the piston skirt are near the top and bottom dead centres with very low sliding velocities resulting in poor lubrication. In order to experimentally

a)

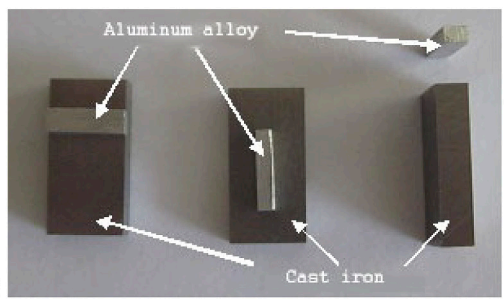

Figure 2: Specimens used in the tribometer tests (a) and SRV4 tribometer (b). 
simulate this situation, normal loads of 25 to $125 \mathrm{~N}$ were chosen (corresponding to nominal contact pressure between 1.5 and $7.5 \mathrm{MPa}$ ) and the sliding speed was varied with $0.1 \mathrm{~m} / \mathrm{s}$ increments from 0.1 to $0.3 \mathrm{~m} / \mathrm{s}$. Similar to the conditions in the engine, the experiments were performed at a temperature of $100^{\circ} \mathrm{C}$.

The contact geometry was chosen in accordance with ideal operation conditions of the real application: block-on-plate with parallel axes of the two interacting bodies. Therefore, the phenomenon of piston slap, where the piston bounces from one side of the liner wall to the other, which is the result of an imperfect alignment of the cylinder liner and the piston axes, is not simulated in this experimental set-up.

The SRV4 tribometer is equipped with sensors for friction and wear measurements, exhibiting the necessary time resolution to determine the variation of the friction coefficient even within one stroke cycle. Using the measurement results, it is possible to calculate the coefficient of friction energy accumulation.

A special feature of the investigated contact interface is the significant difference in the mechanical material properties of the interacting bodies (see Table 1). For example, cast iron has much higher values of hardness and tensile strength than the tested aluminium alloy. Therefore, it is clear that wear rates of the aluminium alloy are much higher compared with the values of the cast iron specimens. For the chosen operation conditions, it turned out that the wear of the cast iron was in the order of the measurement uncertainty $(<0.1 \mu \mathrm{m})$, so it was decided to neglect the contribution of cast iron to the total wear rate and focus just on the higher values of the aluminium alloy.

In order to determine the stress $\sigma$ - constant because of the low normal force - the Brinell hardness is chosen. For low contact pressures, a direct proportionality between normal force and real contact area exists [10]. So the Brinell hardness, converted to the unit MPa, can be used for the stress $\sigma$ [13]. For aluminium, the Brinell hardness - and therefore also $\sigma-$ has a value of $314 \mathrm{MPa}$.

To determine the quantity of linear integral intensity of wear, the focus was put on the steady-state wear regime after the running-in phase of the interface.

\subsection{Results}

In the first wear model discussed above, the coefficient of accumulation of friction energy is a pivotal element needed to determine wear heights in a tribologically stressed system. Because of its definition, it is difficult to measure in an experimental set-up. But it is easy to define $\rho$ as a function of determinable quantities using eqn (15)

Table 1: Selected mechanical properties of the tested specimens.

\begin{tabular}{lcccc}
\hline Material properties & Symbol & Dimension & Aluminium alloy & Cast iron \\
\hline Young's modulus & $E$ & $\mathrm{GPa}$ & 102 & 172 \\
Poisson's ratio & $v$ & - & 0.34 & 0.28 \\
Brinell hardness & $H_{\mathrm{B}}$ & - & 32 & 184 \\
Compressive strength limit & $\sigma_{\mathrm{C}}$ & $\mathrm{MPa}$ & 190 & 294 \\
True fracture stress & $\sigma_{\mathrm{B}}$ & $\mathrm{MPa}$ & 561 & 1275 \\
Yield stress & $\sigma_{\mathrm{T}}$ & $\mathrm{MPa}$ & 152 & 200 \\
Mass density & $\rho$ & $\mathrm{kg} / \mathrm{m}^{3}$ & 2725 & 7845 \\
\hline
\end{tabular}




$$
\rho=\frac{I_{\mathrm{h}} \sigma_{\mathrm{B}}^{2}}{2 \mu \sigma E} .
$$

In the following, the evolution of the coefficient of accumulation of friction energy is analysed for various normal loads and sliding speeds, using the linear integral intensity of wear measured for each point.

Figure 3 shows the typical running-in phase with higher deviations, but with no significant dependence on the normal force. The latter effect validates the constant $\sigma$ approach for low normal forces and pressures. The theoretical models discussed earlier can only be applied to the steady-state phase, because the geometry of the surfaces in the tribocontact must be conforming, which is not the case during running-in. For the tribocontact cast iron-aluminium alloy, the result for $\rho$ in the aluminium alloy under the mean sliding velocity of $v=0.3 \mathrm{~m} / \mathrm{s}$ can be given as the mean value of the last three results in Fig. 3 representing the steady-state phase:

$$
\rho=(6.04 \pm 4.32) \times 10^{-11} .
$$

Figure 4 shows the results for a sliding velocity of $v=0.2 \mathrm{~m} / \mathrm{s}$. The test duration was chosen to examine the running-in phase. An interesting result is seen for a normal force of $F_{\mathrm{n}}=75$ $\mathrm{N}$ : two different experiments were done, with results that spread significantly, which is an indication for the irregular situation in the running-in phase. Therefore, some unavoidable errors occur in this phase, caused by small differences in starting conditions, e.g. by the mounting of the specimens. The results for a sliding velocity of $v=0.1 \mathrm{~m} / \mathrm{s}$ are shown in Fig. 5. These results are similar to those with a sliding velocity of $v=0.2 \mathrm{~m} / \mathrm{s}$.

An exponential fit over the coefficient of accumulation of friction energy for all normal loads was done for each of the three sliding velocities (which were shown separately in Figs 3-5) and are shown in Fig. 6.

The decrease of the absolute values of the coefficient of accumulation of friction energy with increasing sliding speed is in good accordance to the lubrication regime. It must be mentioned that the coefficient of accumulation of friction energy is plotted against operating time and not against the number of load cycles.

\section{rho at $v=0,3 \mathrm{~m} / \mathrm{s}$}

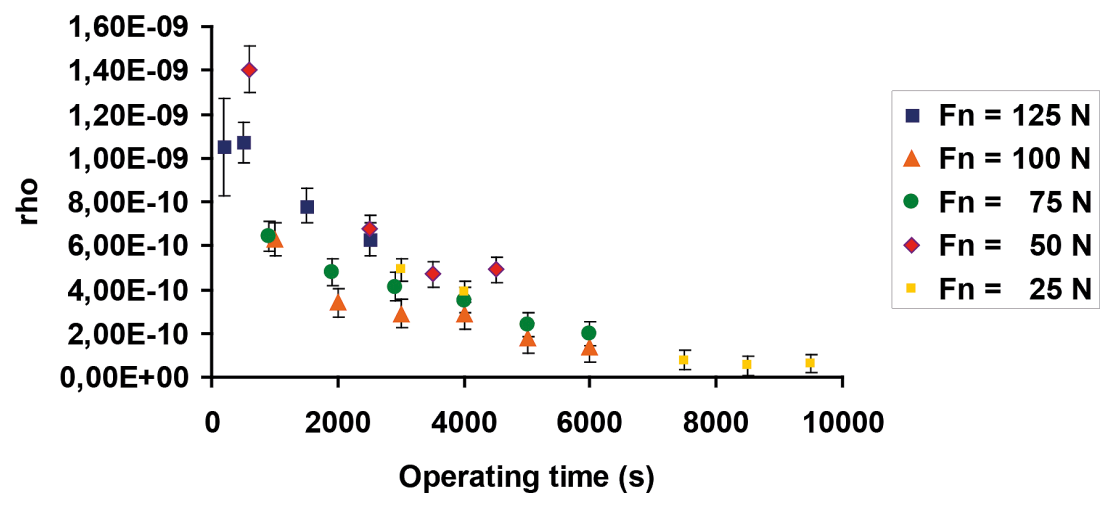

Figure 3: Evolution of the coefficient of accumulation of friction energy $\rho$ over the duration of the experiment for various normal forces $F_{\mathrm{n}}$ at a mean sliding velocity of $0.3 \mathrm{~m} / \mathrm{s}$. 


\section{rho at $v=0,2 \mathrm{~m} / \mathrm{s}$}

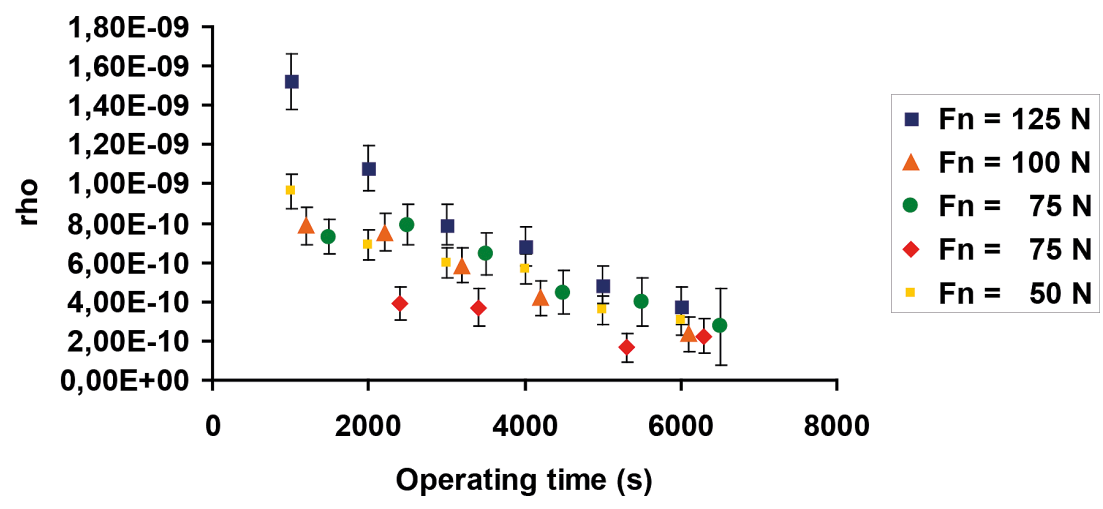

Figure 4: Evolution of the coefficient of accumulation of friction energy $\rho$ over the duration of the experiment for various normal forces $F_{\mathrm{n}}$ at a mean sliding velocity of $0.2 \mathrm{~m} / \mathrm{s}$.

\section{rho at $v=0,1 \mathrm{~m} / \mathrm{s}$}

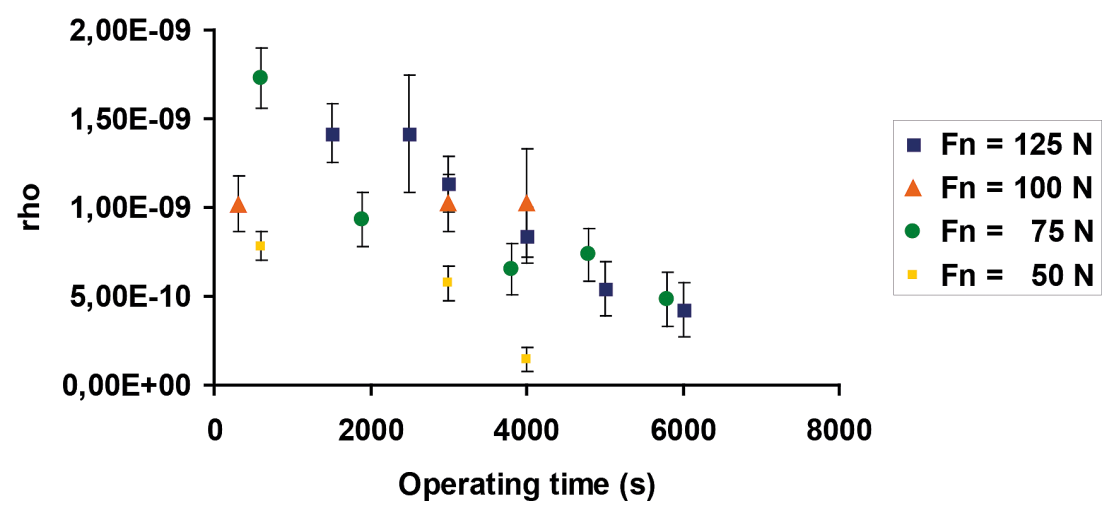

Figure 5: Evolution of the coefficient of accumulation of friction energy $\rho$ over the duration of the experiment for various normal forces $F_{\mathrm{n}}$ at a mean sliding velocity of $0.1 \mathrm{~m} / \mathrm{s}$.

The error bars in Figs 3-5 represent the standard deviation d $\rho$, calculated with the Gaussian error propagation law

$$
\mathrm{d} \rho=\frac{\sigma_{\mathrm{B}}^{2}}{2 \mu E \sigma} \sqrt{d I_{h}^{2}+I_{h}^{2}\left[\left(\frac{d \sigma}{\sigma}\right)^{2}+\left(\frac{d \mu}{\mu}\right)^{2}\right]}
$$

using $\mathrm{d} \mu=0.005$ for the standard deviation of the coefficient of friction $\mu$ (based on statistical scattering of the measurement results), $\mathrm{d} \sigma=15.7 \mathrm{MPa}$ for the standard deviation of the real stress $\sigma$ (estimated as $5 \%$ of $\sigma$ ) and $\mathrm{d} h=0.5 \mu \mathrm{m}$ for the standard deviation of the wear height. The standard deviations for all other parameters are assumed to be negligible. 


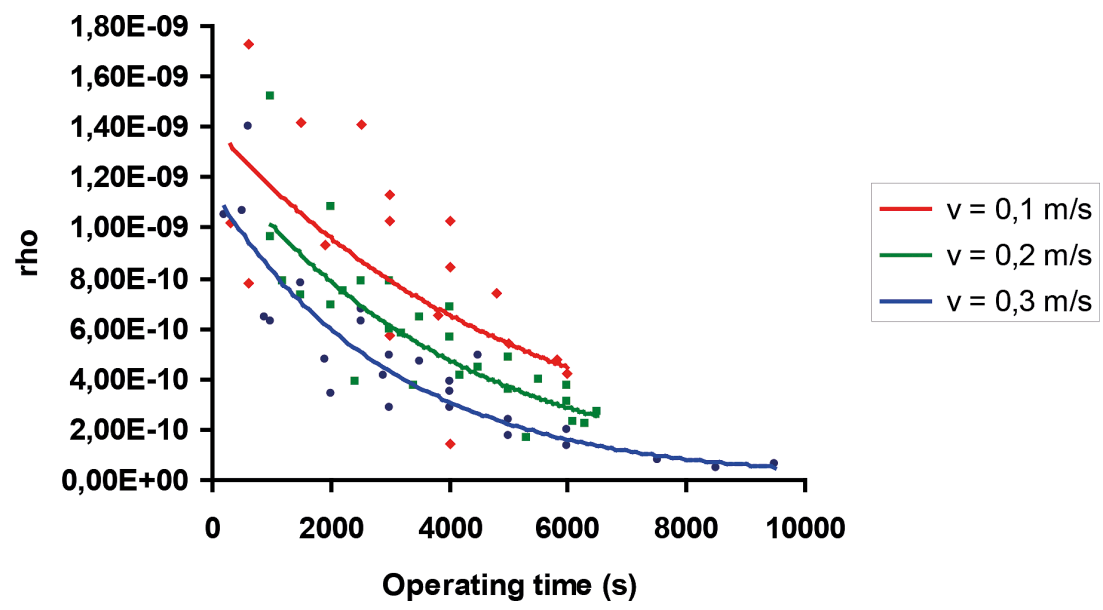

Figure 6: Exponential fit of the coefficient of accumulation of friction energy $\rho$ over various normal forces $F_{\text {n }}$, for mean sliding velocities of $0.1,0.2$ and $0.3 \mathrm{~m} / \mathrm{s}$.

\subsection{Wear phenomena and S-N (Wöhler) curve}

The Wöhler curve shows the behaviour of a material subjected to periodically changing stress loads. In other words, the Wöhler curve uses a known stress amplitude to estimate the number of load cycles until the material breaks off. Therefore, this is an experimental approach to characterise fatigue behaviour. The reason for breaking off after a distinct number of load cycles is a successive accumulation of lattice defects in the material. These are exactly like the ideas behind the theory using the energy accumulation in the boundary layer consisting of asperities to describe wear. Therefore, a similar behaviour with respect to the number of load cycles should be observed when using the same materials. The asperities in the contact zone are stressed with periodic load cycles during the tribological experiment.

To compare the two approaches, the number of necessary macroscopic strokes $n_{\mathrm{M}}$ should be calculated with eqn (23) using the quantities of Table 1 as well as

$$
\begin{aligned}
& \eta_{\mathrm{As}}=12.48 \text { asperities } / \mu \mathrm{m}^{2} \text { (mean value taken from literature [14]) } \\
& \rho=6.04 \cdot 10^{-11} \text { (calculated for the steady-state phase in Fig. 3, eqn (29)) } \\
& F_{\mathrm{n}}=25 \mathrm{~N} \text { (Fig. 3), }
\end{aligned}
$$

which leads to $n_{\mathrm{M}} \sim 53,200$ macroscopic strokes. To compare this number with an S-N curve, this result must be converted to load cycles. A load cycle using the tribometer described above consists of two strokes (one left and one right). The number of macroscopic load cycles $n_{\mathrm{LC}}$ is the half of $n_{\mathrm{M}}$ and, therefore:

$$
n_{\mathrm{LC}}=26600 \text { cycles or } \approx(2.7 \pm 1.9) \times 10^{4} \text { cycles }
$$

$\sigma$ is used to estimate the bending stress of a Wöhler experiment of an asperity. Two S-N curves for aluminium and an aluminium alloy, as well as the calculated $n_{\mathrm{LC}}$, are shown in Fig. 7.

Using the energy-based model discussed in Section 2, it is possible to explain the production of wear with the fatigue processes of the asperities in a tribologically stressed zone. But it should be noted that this model is theoretically valid and experimentally tested only for the 


\section{S - N curve (Wöhler) for Al and Al alloy}

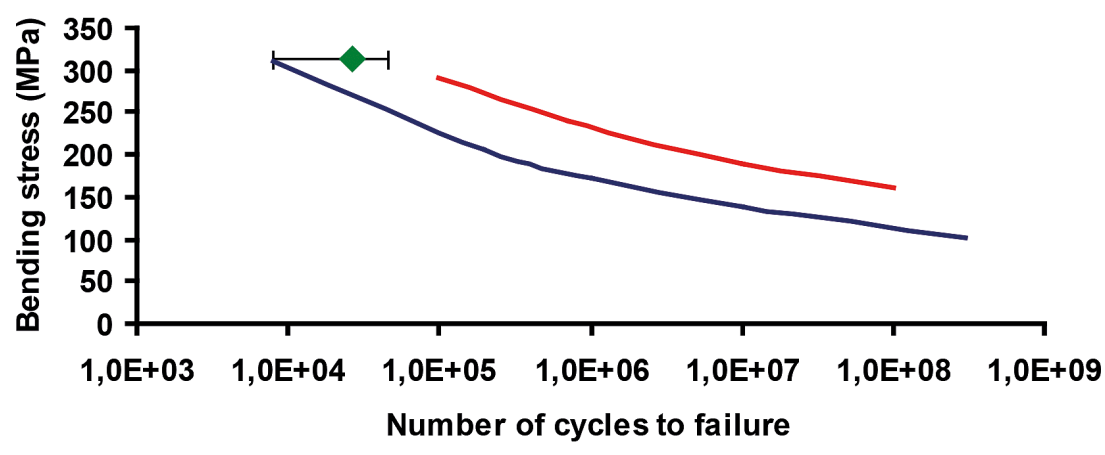

$$
\text { asperity } \longrightarrow \mathrm{Al} \longrightarrow \mathrm{Al} \text { alloy }
$$

Figure 7: S-N curves (Wöhler curve) for aluminium and an aluminium alloy. The green symbol indicates the result of experimental number of critical load cycles for a Wöhler experiment with an asperity of the tribological interface zone.

steady-state phase and a tribocontact consisting of a hard and a soft partner. Only the wear released by the soft partner is taken into consideration.

\section{DISCUSSION AND COMPARISON OF THE WEAR MODELS}

As mentioned in Section 1, the different power laws will not lead to a deeper understanding of wear phenomena. However, the wear coefficient $K_{\omega}$ of the power law in eqn (27) can be analysed further with the results of the energy model. To compare this equation with eqn (16), the latter has to be modified, using the ratio of the real to nominal contact areas so as to include the nominal contact pressure $p_{\mathrm{A}}$ instead of the stress $\sigma$ :

$$
\theta=\frac{2 \mu E A_{\mathrm{N}} p_{\mathrm{A}} v}{\sigma_{\mathrm{B}}^{2} A_{\mathrm{R}}} \rho .
$$

Letting $\alpha$ and $\beta$ equal 1, the comparison with eqn (31) leads to

$$
K_{\omega}=\frac{2 \mu E A_{\mathrm{N}}}{\sigma_{\mathrm{B}}^{2} A_{\mathrm{R}}} \rho
$$

or, without $A_{\mathrm{R} \text {, }}$

$$
K_{\omega}=\frac{2 \mu E A_{\mathrm{N}} \sigma}{\sigma_{\mathrm{B}}^{2} F_{\mathrm{n}}} \rho .
$$

For the steady-state phase and contact conditions of the experimental set-up described in Section 3:

$$
\begin{aligned}
& \mu=0.19 \\
& F_{\mathrm{n}}=25 \mathrm{~N} \\
& A_{\mathrm{N}}=15 \mathrm{~mm}^{2}
\end{aligned}
$$


and the constant $K_{\omega}$ is calculated to be: $K_{\omega}=1.4 \times 10^{-15}\left(\mathrm{~Pa}^{-1}\right)$. According to literature [11], the modified dimensionless constant

$$
K=K_{\omega} \sigma
$$

describes the severity of wear. Typically, $K \sim 10^{-8}$ falls into the category of 'mild' wear, whereas $K \sim 10^{-2}$ would be 'severe' wear. $K=4.4 \times 10^{-7}$ for the example used in the calculations above, which therefore falls into the range of relatively 'mild' wear.

\section{CONCLUSION}

It is possible to explain macroscopic fatigue problems and the production of wear using the same physical principle. The successive accumulation of lattice defects within a macroscopic element described by the $\mathrm{S}-\mathrm{N}$ curve (Wöhler) is comparable to the accumulation in microscopic asperities describing the interacting surface geometry of a tribocontact.

\section{ACKNOWLEDGMENTS}

The experimental part was carried out at financial supporting of Ministry of Education and Science of Russian Federation for realization of applied researches by lot number 2014-14579-0109 with unique identifier of applied scientific researches (project) RFMEFI57714X0102. Data evaluation and discussions of the work were funded by the Austrian COMET-Program (Project K2, XTribology, no. 824187/849109) and carried out at the "Excellence Centre of Tribology" as well as at the South Ural State University which is recipient of subsidy, agreement number 14.577.21.0102.

\section{REFERENCES}

[1] Meng, H.C., Wear modelling: evaluation and categorization of wear models, $\mathrm{PhD}$ Thesis, University of Michigan: Ann Arbor, MI, 1994.

[2] Hsu, S.M., Shen, M.C. \& Ruff, A.W., Wear prediction for metals. Tribology International, 30(5), pp. 377-383, 1997. ISSN 0301-679X. doi: http://dx.doi.org/10.1016/ s0301-679x(96)00067-9

[3] Meng, H.C. \& Ludema, K.C., Wear models and predictive equations: their form and content, Wear, 181-183(2), pp. 443-457, 1995. ISSN 0043-1648. doi: http://dx.doi. org/10.1016/0043-1648(95)90158-2

[4] Fleischer, G., Energetische Methode der Bestimmung des Verschleißes. Schmierungstechnik, Band 4, pp. 9-12, 1973.

[5] Lazarev, V., Gavrlov, K., Doikin, A., Vorlaufer, G. \& Sequard-Base, J., Estimation of the tribotechnical parameters of the "piston skirt-cylinder liner" contact interface from an IC-engine for decreasing the mechanical losses. WIT Transactions on Ecology and the Environment, 190 (1), pp. 625-635, 2014. doi: http://dx.doi.org/10.2495/eq140601

[6] Ye, Z., Zhang, C., Wang, Y., Cheng, H.S., Tung, S., Wang, Q.J. \& He, X., An experimental investigation of piston skirt scuffing: a piston scuffing apparatus, experiments, and scuffing mechanism analyses. Wear, 257(1-2), pp. 8-31, 2004. ISSN 0043-1648. doi: http://dx.doi.org/10.1016/s0043-1648(03)00538-6

[7] OICA. Production statistics. http://www.oica.net/category/production-statistics/ (accessed on 9 October 2014).

[8] Fouvry, S. \& Kapsa, P., An energy description of hard coating wear mechanisms. Surface and Coatings Technology, 138(2-3), pp. 141-148, 2001. ISSN 0257-8972. doi: http://dx.doi.org/10.1016/s0257-8972(00)01161-0 
[9] Fouvry, S., Liskiewicz, T., Kapsa, P., Hannel, S. \& Sauger, E., An energy description of wear mechanisms and its applications to oscillating sliding contacts. Wear, 255(1-6), pp. 287-298, 2003. ISSN 0043-1648, http://dx.doi.org/10.1016/S0043-1648(03)00117-0. doi: http://dx.doi.org/10.1016/s0043-1648(03)00117-0

[10] Greenwood, J. \& Williamson, J., Contact of nominally flat surfaces, Proceedings of the Royal Society A, 295(1442), pp. 300-319, 1966. doi.org/10.1098/rspa.1966.0242. doi: http://dx.doi.org/10.1098/rspa.1966.0242

[11] Archard, J. \& Hirst, W., The wear of metals under unlubricated conditions. Proceedings of Royal Society London A, 236(1206), pp. 397-410, 1956. doi: http://dx.doi. org/10.1098/rspa.1956.0144

[12] Goryacheva, I., Contact Mechanics in Tribology, Kluwer: Dordrecht, 1998. ISBN 9780792352570. doi: http://dx.doi.org/10.1007/978-94-015-9048-8

[13] Jisa, R., Tribologische Wechselwirkungen von selbstschmierenden Gleitelementen basierend auf Kupferlegierungen und Graphit-Öl-Schmierstoffen. PhD Thesis, Vienna University of Technology, 2007.

[14] Yeo, C., Katta, R., Lee, J. \& Polycarpou, A., Effect of asperity interactions on rough surface elastic contact behaviour: hard film on soft substrate. Tribology International, 43(8), pp. 1438-1448, 2010. ISSN 0301-679X. doi: http://dx.doi.org/10.1016/j.triboint.2010.01.021 